\title{
Prevalence of Hepatitis C and B Virus Infection in Patients with Idiopathic Dilated Cardiomyopathy in Brazil: A Pilot Study
}

\author{
Francisco J.F.B. Reis ${ }^{1}$, Mateus Viana ${ }^{2}$, Manoela Oliveira ${ }^{2}$, Tiago A. Sousa ${ }^{2}$ and Raimundo Paraná ${ }^{3}$ \\ ${ }^{1}$ Post-graduation Course in Medicine and Public Health of the Bahiana School of Medicine and Public Health; ${ }^{2}$ Academician of the Bahia \\ Federal University Medical School (UFBA); ${ }^{3}$ PhD in Hepathology and Professor of Federal University of Bahia (UFBA); Salvador, BA, Brazil
}

The idiopathic dilated cardiomyopathy (IDMC) is a disease of the cardiac muscle characterized by systolic dilation and/or dysfunction of one or both ventricles, symptoms of congestive heart failure and risk of early death. Several studies in animal models and in humans have supported the hypothesis of the viral infection as initial event in the immunopathogenesis of the ventricular dilation. The objective of this study was to correlate the presence of hepatitis $\mathrm{C}$ virus chronic infection and idiopathic dilated cardiomyopathy, comparing samples of cases with IDCM with paired controls with Chagas-type specific dilated cardiomyopathy and ischemic-type specific dilated cardiomyopathy. Only $2.9 \%$ (1/34) of IDCM patients were HCV carriers, which strongly argue against this hypothesis. Therefore, based on our results, there is no justification for investigating $\mathrm{HCV}$ in patients with idiopathic dilated cardiomyopathy diagnosis.

Key-Words: Idiopathic dilated cardiomyopathy, hepatitis $\mathrm{C}$ virus, myocarditis, heart failure.

Cardiomyopathies are defined as myocardium diseases associated with alteration in the cardiac function. Cardiomyopathies are classified into dilated cardiomyopathy, hypertrophic cardiomyopathy, restrictive cardiomyopathy and arrhythmogenic right ventricular dysplasia [1]. The idiopathic dilated cardiomyopathy (IDNC) is a disease of the cardiac muscle characterized by systolic dilation and/or dysfunction of one or both ventricles, frequently presenting symptoms of congestive heart failure and risk of early death [2,3]. Among the dilated cardiomyopathies, the IDCM has been reported as the one presenting the highest frequency, followed by viral myocarditis and coronary arterial disease with $46.5 \%, 12 \%$ and $11 \%$, respectively [4]. In Brazil, the IDCM is an unusual cause of CHF [5], but an important cause of $\mathrm{CHF}$ in developed countries, involving $25 \%$ of all patients with this syndrome [6].

The myocarditis is frequently related to the IDCM pathogenesis [7] and the infectious agents, especially viral agents, are the most important causes. The enteroviruses and the Coxsakie B3 and B4 viruses are the most widely related with myocarditis. Recently, other viral agents have emerged in the myocarditis pathogenesis, among them, the hepatitis $\mathrm{C}$ virus has been more frequently described in Japan. The first work that analyzed the presence of the viral RNA in the myocardium of patients with dilated cardiomyopathy (IDCM) was published in 1995 in Japan and reported that 6/36 (16.7\%) of patients were $\mathrm{HCV}$ infected, while this virus was found in the serum of only 1 out of 40 patients $(2.5 \%, \mathrm{p}<0.05)$ from the control group composed of patients with ischemic cardiopathy [8]. Other studies involving the same group demonstrated

Received on 5 January 2007; revised 30 May 2007.

Address for correspondence: Dr. Francisco José Farias Borges do Reis. Rua Quintino de Carvalho, $\mathrm{n}^{\mathrm{o}}$ 113, Apt $^{\mathrm{o}}$ 301, Jardim Apipema. Zip code: 40155-280. Salvador, Bahia, Brazil. E-mail: fbreis@ cardiol.br. Phone: 71 3332-5870 / 8876-5870.

The Brazilian Journal of Infectious Diseases

2007;11(3):318-321. (C) 2007 by The Brazilian Journal of Infectious Diseases and Contexto Publishing. All rights reserved. similar relation between $\mathrm{HCV}$ and dilated cardiomyopathy, particularly the IDCM [9]. Despite the favorable evidences of the HCV participation in the genesis of cardiomyopathies and myocarditis, the literature presents opposite data. These data support the necessity of a careful evaluation due to the possibility of false positives using the PCR technique in these studies and also the detection of RNA of the HCV is due to infected lymphocytes actively infiltrating the myocardium in the myocarditis process rather than the direct myocytes infection [10].

However, the etiological role of the HCV in the idiopathic dilated cardiomyopathy remains controversial, justifying the performance of new studies in relation to this subject with the objective of investigating if regional factors are influencing this association.

The objective of this study was to evaluate the prevalence of HCV infection among patients with dilated myocardiopathy as well as the existence of association between HCV and idiopathic dilated cardiomyopathy.

\section{Materials and Methods}

In the period from January 2002 to July 2004, all patients with idiopathic dilated cardiomyopathy were identified through the active search of data from all patients enrolled in the Edgard Santos General Hospital cardiomyopathy clinics.

The idiopathic dilated cardiomyopathy was identified as the dilated cardiomyopathy presenting no specific etiological cause - negative serology for Chagas' heart disease, patients older than 45 years of age and with coronary angiographic study with no obstruction above $50 \%$, patient should not have been submitted to chemotherapy for neoplasia, present no history of alcoholism and absence of arterial hypertension degrees II or III according to classification proposed by the VI "Joint International Committee" for the diagnosis and treatment of arterial hypertension.

Concomitantly, we have selected individuals attended in the hospital with no history of alcoholism, ischemic cardiopathy, systemic arterial hypertension degrees II and III, 
not submitted to chemotherapy treatment nor with positive serology for Chagas heart disease as the control group of patients with ischemic cardiopathy.

The control group with Chagas heart disease was selected through serology with the ELISA test with the exclusion of other conditions that could lead to idiopathic dilated cardiomyopathy. Patients from the group mentioned above and with coronary disease had clinical and angiographic corroboration of their cardiopathy.

The anti-HCV was determined by Elisa tests using commercial Kits from Roche (Basel, Switzerland). The HCVRNA was performed using Amplicor from Roche (Basel, Switzerland).

Once the HCV infection was used as dependent variable, two methods were used for the HCV infection investigation: serological analysis and viral RNA research (in case of positive serology). Gender, age, cardiomyopathy etiology, functional class, time of disease and the echodopplercardiographic parameters were analyzed as independent variables.

\section{Statistical Analysis and Ethic Aspects}

The Chi-square and Fisher exact tests were used to compare proportions and the t-Student test was used to compare averages between groups. All analyses were performed through the SPSS program (Statistical Package of Social Sciences) version 9.0. All hypotheses tests were bicaudal with $\mathrm{p}$ value lower than 0.05 , indicating statistical significance.

This research project was sent for appreciation and received approval from the Ethics Commission of the Professor Edgard Santos General Hospital. During the data collection, all procedures were explained to patients who signed the consent term in order to participate in this study.

\section{Results}

One hundred and five patients were studied, among them 34 with idiopathic dilated cardiomyopathy diagnosis, 32 with ischemic cardiomyopathy and 39 with cardiomyopathy due to Chagas heart disease. The age ranged from 20 to 77 years (average age of 46 years) and the male gender was the most frequent (Table 1).

The majority of patients from the three groups were composed of retired individuals who denied past of sexually transmissible disease. All patients reported the absence of past hepatitis, not having shared syringes or used glass syringes, no past of blood transfusion, absence of family hepatic disease and no past work in health services (Table 1). In relation to medications used, most patients from all groups were making use of angiotensin-converting enzyme inhibitors (ACEI), digital and diuretic. Beta-blockers were used by most components of the idiopathic and ischemic groups $(64.7 \%$ and $68.8 \%$, respectively) and used by only $12.8 \%$ of components of the Chagas heart disease group (Table 2).

The clinical data in relation to the functional class were homogeneous between groups, and most patients presented functional class I or II. A variation between groups was observed in relation to the time of cardiovascular symptoms, which was longer in the Chagas heart disease group (Table 2). No significant differences were observed in the echocardiographic data in relation to the ejection fraction and diastolic diameter of the left ventricle in all groups. However, the alterations on the ventricular kinetics were higher in the ischemic group (Table 2).

With regard to the anti-HCV serological data, only $1 / 34$ $(2.9 \%)$ of patients with IDMC presented positive result. All other patients had negative results for anti-HCV and $\mathrm{HCV}$ RNA. No one were either positive for HBsAg (Table 2).

The patient who presented positive anti-HCV serology was male, 45 years old, caucasian, in FC I/IV NYHA, presented ejection fraction through the Simpson method of $12 \%$ and shortening fraction of $13.04 \%$ with diastolic diameter of 69 $\mathrm{mm}$, final systolic diameter of $60 \mathrm{mM}$ and restrictive-type diastolic standard (evidencing severe involvement of the left ventricle). This patient had no evidence of possible origin of the exposure to $\mathrm{HCV}$.

\section{Discussion}

The hypothesis that viral infections are related to cardiomyopathies is based on studies that used techniques and methods of molecular biology [11]. Some studies support that viral infections would be related to the development of myocarditis and idiopathic dilated cardiomyopathy (IDCM), suggesting that the IDCM would be a result of a previous subclinical myocarditis. The viral infection has also been related with restrictive cardiomyopathy, as demonstrated by Tschope, Bock C-T et al. in study with 70 patients that showed strong association between parvovirus B19 and isolated left ventricular diastolic dysfunction [12].

Besides enterovirus, that has so far been the infectious agent most accepted as etiological factor of the myocarditis and IDCM, there is a wide variety of other viruses being considered as etiological agents [13]. Kuhl Uwe et al. in other study performed in Germany, analyzed samples of endocardial biopsies of 245 patients presenting systolic dysfunction detected through echocardiogram. Viral genome was found in 165 out of the 245 patients $(67.4 \%) ; 126$ of them $(51.4 \%)$ with parvovirus B19, 53 (21.6\%) with human herpes virus-6, 23 $(9.4 \%)$ with enterovirus and in lower percentage adenovirus, Epstein Barr virus and cytomegalovirus. In 45 patients (27.3\%), multiple viral infections were found, suggesting that this infection may play a role in the IDCM pathogenesis far more frequent than the expected so far [14].

Some studies suggest association between $\mathrm{HCV}$ and IDCM. Matsumori et al demonstrated anti-HCV antibodies in the serum of $6 / 36$ patients $(16.7 \%)$ with IDCM and thus, suggested that the hepatitis $\mathrm{C}$ virus (HCV) would be contributing for its development [10]. Similarly, Okabe et al studied the association between HCV infection and active chronic myocarditis, a variant form of myocarditis characterized by numerous lymphocyte agglomerates and myocardial cell lesion in 3 patients [9]. 
Table 1. Demographic characteristics and exposition to hepatitis $\mathrm{C}$ virus in patients with heart failure of different etiologies (idiopathic, ischemic and Chagas heart disease)

\begin{tabular}{|c|c|c|c|c|}
\hline & Group I & Group II & Group III & Pvalue \\
\hline & Idiopathic (n=34) & Ischemic $(\mathrm{n}=32)$ & Chagas $(n=39)$ & \\
\hline Age & $44 \pm 11.5$ & $59 \pm 9.5$ & $47 \pm 11.3$ & 0.001 \\
\hline Male gender & $23(67.6 \%)$ & $25(78.1 \%)$ & $25(64.1 \%)$ & 0.43 \\
\hline Non-white race & $25(73.5 \%)$ & $22(68.7 \%)$ & $34(87.2 \%)$ & 0.36 \\
\hline Marital status-Married & $21(61.8 \%)$ & $22(68.8 \%)$ & $23(59.0 \%)$ & 0.25 \\
\hline Use of shared or glass syringes & $6(17.6 \%)$ & $8(25.0 \%)$ & $6(15.4 \%)$ & 0.31 \\
\hline Blood transfusions & $1(2.9 \%)$ & $11(34.4 \%)$ & $4(10.3 \%)$ & 0.05 \\
\hline Use of condom & $14(41.2 \%)$ & $7(21.9 \%)$ & $12(30.8 \%)$ & 0.51 \\
\hline Family hepatic disease & $9(26.5 \%)$ & $5(15.6 \%)$ & $12(30.8 \%)$ & 0.34 \\
\hline STD & $10(29.4 \%)$ & $11(34.4 \%)$ & $9(23.1 \%)$ & 0.57 \\
\hline Worked in health services & $4(11.8 \%)$ & $3(9.4 \%)$ & $6(15.4 \%)$ & 0.74 \\
\hline Surgical procedures & $18(52.9 \%)$ & $22(68.8 \%)$ & $20(51.3 \%)$ & 0.28 \\
\hline Invasive procedures & $19(55.9 \%)$ & $32(100 \%)$ & $21(53.8 \%)$ & 0.0001 \\
\hline
\end{tabular}

$\dagger$ Data are presented as average \pm standard deviation - quantitative variables - or absolute number (valid percentage) - categorical variables. SDT=sexually transmissible disease.

Table 2. Medications in use, functional class according to the NYHA, echocardiographic data and serology of patients with heart failure from the three different groups (idiopathic, ischemic and Chagas heart disease)

\begin{tabular}{lcccc}
\hline & Group I & Group II & Group III & Pvalue \\
\cline { 2 - 4 } & Idiopathic (n=34) & Ischemic (n=32) & Chagas (n=39) & \\
\hline Digital & $73.5 \%$ & $68.8 \%$ & $64.1 \%$ & -- \\
ACEI & $91.2 \%$ & $78.1 \%$ & $82.1 \%$ & -- \\
B-blocker & $64.7 \%$ & $68.8 \%$ & $12.8 \%$ & -- \\
Blocker AT1 & $8.8 \%$ & $18.8 \%$ & $10.3 \%$ & -- \\
Furosemide & $58.8 \%$ & $75 \%$ & $74.4 \%$ & -- \\
Spironolactone & $64.7 \%$ & $37.5 \%$ & $64.1 \%$ & -- \\
FC I or II & $26(76.5 \%)$ & $25(78.2 \%)$ & $28(71.8 \%)$ & -- \\
FC III or IV & $8(23.5 \%)$ & $7(21.8 \%)$ & $11(28.2 \%)$ & -- \\
Time of symptoms & $2.5+2.6$ years & $3.1+3.98$ years & $6.0+5.8$ years & 0.02 \\
EF Simpson \% & $22.0 \pm 9.9$ & $24.9 \pm 10.2$ & $23.9 \pm 9.5$ & 0.51 \\
FDD(mm) & $70.2 \pm 11.1$ & $69.7 \pm 6.5$ & $67.2 \pm 10.3$ & 0.37 \\
Segmental Alteration & $0 \%$ & $29(90.6 \%)$ & $10(25.6 \%)$ & 0.0001 \\
HBsAg & $0 \%$ & $0 \%$ & $0 \%$ & - \\
Anti-HCV & $1(2.9 \%)$ & $0 \%$ & $0 \%$ & 0.337 \\
\hline
\end{tabular}

$\dagger$ Data are presented as average \pm standard deviation - quantitative variables - or absolute number (valid percentage) - categorical variables. ACEI=angiotensin-converting enzyme inhibitors. Blocker AT1=angiotensin II subtype 1 receptor blocker. FC=functional class. $\mathrm{EF}=$ ejection fraction. $\mathrm{FDD}=$ final diastolic diameter.

Using the genomic analysis for the detection of RNA of the HCV, these authors concluded that the HCV might replicate in the myocardial tissue and thus, contribute for the development of the chronic myocarditis $[9,10]$. In another study involving individuals with hypertrophic cardiomyopathy, which is a disease associated with a genetic alteration, the RNA of the HCV was also found in the myocardial tissue of 3 patients [8].

Despite these studies [8-10], there is no agreement that the hepatitis $\mathrm{C}$ virus could effectively replicate in the myocardium or that it could be related to the IDCM pathogenesis. A reasonable explanation for the detection of the viral genome in the myocardial cells is that the RNA amplification may simply be reflecting the presence of viral material of infected neighbor cells or plasma. Thus, all patients with IDCM reported so far, in which the RNA of the HCV was identified in the myocardium, were also positive for RNA of the HCV in the serum. These patients usually have several hospitalizations with invasive procedures, so they are particularly exposed to nosocomial HCV transmission. In addition, the $\mathrm{HCV}$ replication in the myocardium can only be verified through other laboratorial techniques such as in situ hybridization [10]. Moreover, the negative helix of the RNA should be found in order to certify the replicating status. 
Corroborating these data, Dalekos and colleagues found no patient with chronic HCV infection with clinical or subclinical signs of IDCM based on anamnesis or laboratorial data and yet, none of the patients with dilated cardiomyopathy diagnosis was positive for anti-HCV or viral antibody in the test for the detection of RNA of the HCV [15]. These results are in agreement with ours, although there were markable methodological differences between both studies.

In our study, 105 patients with diagnosis of idiopathic dilated cardiomyopathy, ischemic cardiomyopathy and cardiomyopathy due to Chagas heart disease were enrolled. We found no evidence supporting any association between HCV infection and the IDCM development. Although the development of the dilated cardiomyopathy due to HCVinduced myocarditis cannot be definitely excluded by studies that analyzed blood samples only, the absence of viral markers in these patients tends to reject this hypothesis at least in our population.

A reasonable explanation for the discrepancy of our findings when compared with those reported by Japanese researchers would be the prevalence of HCV infection in this population, where most part of the studies was conducted. On the other hand, evolutive clinical forms distinct from the $\mathrm{HCV}$ infection were described in different countries and interestingly, more severe forms were observed in Japan [16]. Data collected from blood donations in Brazil revealed a positive index of $1.2 \%$ [17], while the same research involving Japanese blood donors revealed a prevalence of $1.65 \%$ in individuals from 45 to 49 years of age and of $2.41 \%$ in those between 55 and 59 years old (information from the Japanese Blood Donation Center, 1990). The contrasting results may be explained, at least in part, by regional particularities. Moreover, the HCV extra-hepatic manifestations are a result of the interaction between the host and the virus, where the host genetics background may play important role.

The sample of patients with idiopathic dilated cardiomyopathy in the present work was composed of the entire population of patients enrolled in the HUPES (University Hospital of Bahia, Brazil) cardiomyopathy clinics; therefore, there is no possibility of extracting a subsample from the studied population. These patients were well investigated for other etiologies such as hypertensive and especially ischemic (most patients presented ischemic tests and coronary angiography that did not demonstrate myocardial ischemia of possible atherosclerotic etiology); therefore, with a very probable diagnosis of idiopathic dilated cardiomyopathy. The control group composed of patients with ischemic-type dilated cardiomyopathy was selected due to the high number of invasive procedures conducted in these patients, thus representing a high exposure to HCV. The control group composed of patients with Chagas-type dilated cardiomyopathy was also selected due to the infectious component of a specific agent (Trypanosome cruzi) in the cardiomyopathy physiopathology.

\section{Conclusion}

The present study does not establish association between HCV infection and idiopathic dilated cardiomyopathy. Therefore, there is no justification for routine investigation of $\mathrm{HCV}$ in patients with idiopathic dilated cardiomyopathy. We consider, however, that this point is not fully clear and deserve further case-control studies with large number of patients.

\section{References}

1. Richardson P., Mckenna W., Bristow M. Report of the 1995 World Health Organization International Society and Federation of Cardiology - Task Force on the Definition and Classification of Cardiomyopathies. Circulation 1996;93(5):841-2.

2. Dec G.W., Fuster V. Idiopathic dilated cardiomyopathy. N Eng J Med 1994; 331(23): 1564-75.

3. Coughlin S.S., Neaton J.D., Sengupta A., Kuller L.H. Predictors of mortality from idiopathic dilated cardiomyopathy in 356222 men screened for the Multiple Risk Factor Intervention Trial. Am J Epidemiol 1994;139(2):166-72.

4. Kasper E.K., Agema W.R., Hutchins G.M., et al. The Cause of idiopathic dilated cardiomyopathy: a clinicopathologic review of 673 consecutive patients. J Am Coll Cardiol 1994;23(3):591-2.

5. Adams K.F., Zannad F. Clinical definition an epidemiology of advanced heart failure. Am Heart J 1998;135(6 Pt $2 \mathrm{Su})$ : S204-15

6. Bestetti R.B., Muccillo G. Clinical course of Chagas heart disease: a comparison with dilated cardiomyopathy. Int J Cardiol 1997;60:187-93.

7. Bowles N.E., Richardson P.J., Olsen E.G., Archard L.C. Detection of Coxsackie B virus specific RNA sequences in myocardial biopsy samples from patients with myocarditis and dilated cardiomyopathy. Lancet 1986;1(8490):1120-3.

8. Matsumori A., Matoba Y., Nishio R., et al. Detection of hepatitis $\mathrm{C}$ virus RNA from the heart of patients with hypertrophic cardiomyopathy. Biochem Biophys Res Commun 1996;222(3):678-82.

9. Okabe M., Fukuda K., Arakawa K., et al. Chronic variant of myocarditis associated with hepatitis $\mathrm{C}$ virus infection. Circulation 1997;96(1):22-4.

10. Matsumori A., Matoba Y., Sasayama S. Dilated cardiomyopathy associated with hepatitis $\mathrm{C}$ virus infection. Circulation 1995;92(9):2519-25.

11. Liang T.J., Reherman B., Leonard B., et al. Pathogenesis, Natural History, Treatment and Prevention of Hepatitis C NIH Conference. Annals of Internal Medicine 2000;132(4):296-305.

12. Tschope C., Bock C-T., Kasner N., et al. High prevalence of cardiac parvovirus B19 infection in patients with isolated left ventricular diastolic dysfunction. Circulation 2005;111(7):879-86.

13. Brandão A.B.M., Fuchs S.C., Silva M.A.A., Emer L.F. Diagnóstico da hepatite $\mathrm{C}$ na prática médica: revisão de literatura. Pan Am Health 2001;9(3):161-8.

14. Tschope C., Pauschinger M., Noutsias M., et al. High prevalence of viral genomes and multiple viral infections in the myocardium of adults "idiopathic" left ventricular dysfunction. Circulation 2005; 111(7):887-93.

15. Dalekos G.N., Achenbach K., Christodoulou D., et al. Idiopathic dilated cardiomyopathy: lack of association with hepatitis C virus infection. Heart 1998;80(3):270-5.

16. Grumbach I.M., Heermann K., Figulla H.R. Low prevalence of hepatitis $\mathrm{C}$ virus antibodies and RNA in patients with myocarditis and Dilated cardiomyopathy. Cardiology 1998;90(2):75-8.

17. Saúde em Mapas e Números. Boletim informativo. Hepatites no Brasil. Eurotrials Scientific Consultants 2003; 4: 1-4. Available from: URL: http://www.eurotrials.com/publicacoes/ bolbrpt4.pdf.Eurotrials. 